\title{
The health and economic impacts of obesity
}

\begin{abstract}
The sharp rise in the prevalence of obesity in many countries have raised major global concern. Obesity is broadly preventable and has a complex multifactorial aetiology. The prevalence of obesity is on the rise and has increased by threefold since the mid 70s of last century. Obesity has overwhelming effect on individual's health in terms of worsening morbidity and increased risk of mortality and on public health overall due to its economic burden on the health system. This narrative review utilises many published epidemiological studies that focus on the consequences of obesity in order to provide a framework for the overall cost of obesity in terms of its morbidity, mortality, and economic burden.
\end{abstract}

Volume 10 Issue 4 - 2020

Tahir Omer

Northampton General Hospital, University of Chester, UK

Correspondence: Tahir Omer, Northampton General Hospital, University of Chester, UK, Email tahir.omer@chester.ac.uk

Received: June 21, 2020 | Published: July 14, 2020

Keywords: obesity, weight gain, health promotion, nutrition, public health, diabetes, NAFLD, health economics, economic burden

\section{Introduction}

The sharp rise in the prevalence of obesity in many countries have raised major global concern. Obesity is broadly preventable, and it is caused by complex multifaceted interaction between genetics, environmental and human behaviour factors. ${ }^{1,2}$ The prevalence of obesity has increased by threefold since the mid 70s of last century and If the current global trend continues, it is predicted that over a billion of the world population will be obese in 5 to 10 years. ${ }^{1}$ The World Health organisation (2019) defines obesity as excessive abnormal fat storage in individual's body that leads to adverse health outcome. It is usually measured using Body Mass Index (calculated by dividing the weight in kilograms by the square height in metres). BMI functions as an effective initial screening method to classify obesity rather than a precise diagnostic tool. ${ }^{1}$ Obesity has overwhelming effect on individual's health in terms of worsening morbidity and increased risk of mortality and on public health overall due to its economic burden on the health system. This article utilises several published epidemiological studies that focus on the consequences of obesity in order to provide a framework for the overall cost of obesity in terms of its morbidity, mortality, and economic burden.

\section{The health consequences of obesity}

The health consequences of obesity can generally be divided into 2 groups. Those adverse health outcomes due the metabolic and secretory function of the excessively accumulating adipose tissue and those outcomes secondary to the mass effect of the expanding adipose tissue. ${ }^{3}$ Both effects can lead to increased overall mortality and contribute to the chronicity of morbidities related to obesity. ${ }^{4}$ The metabolic effect of obesity includes the risk of developing type 2 Diabetes, high blood pressure, cardiovascular disease, gallstones and several types of cancer which are more prevalent in obese individuals. On the other hand, the mass effect of accumulating adipose tissue can result in cardiopulmonary disease due to obstructive sleep apnoea, joint disease due to osteoarthritis in addition to the psychological burden and stigma related to obesity. ${ }^{3,4}$

\section{Overall mortality risk}

Overall, obesity is directly related to increased mortality. ${ }^{4} \mathrm{~A}$ large American cohort in 2006 reviewed the risk of death in relation to body weight in over half a million Americans of both genders in their $6^{\text {th }}$ and $7^{\text {th }}$ decades of life. After adjusting relative risks for ethnicity, socioeconomic background, smoking and alcohol consumption and running extra analyses to tackle possible confounders due to established comorbidities, mortality was reported to have increased by more than one fifth in overweight subjects and by 2 - to 3 -fold in the obese category. The study, however, was limited by the fact that the BMI was calculated from self-reported weight and height rather than actual precise measurements.

A prospective cohort study in 2006 in Korea examined the association between body weight and overall mortality over 10-year period. ${ }^{5}$ The cohort included more than a million Koreans from the $4^{\text {th }}$ decade in life up to the $10^{\text {th }}$ decade. Over 80 thousand deaths from any cause in relation to the body-mass index (BMI) were reviewed and it was found that mortality from any cause had a direct association with BMI, irrespective of smoking status. The mortality was lowest among patients with a BMI in the normal range of 23.0 to 24.9. Mortality from cardiovascular disease or cancer was higher among the higher BMI group. Interestingly, their findings support that being on both sides of the extreme whether underweight or overweight increases mortality in both genders.

\section{The metabolic consequences of adiposopathy}

The main causes of premature death in obesity is heart disease related to high blood pressure and Cardiac events. ${ }^{6}$ The risk of hypertension doubles to trebles in both genders with a BMI greater than 25.0 in comparison to individuals with BMI in the normal range. This risk worsens further with BMI greater than 29.0. Much of the increase in the prevalence of heart attacks and strokes noticed in obesity can be due to high blood pressure, diabetes and abnormal lipid metabolism. ${ }^{6,7}$ Moreover, beside the role of obesity as a causative factor and contributor to the chronicity of these conditions, obesity on its own appears to be a direct risk factor for macrovascular disease. ${ }^{7}$ The risk of developing type 2 diabetes is directly related to BMI. This has been recognised since 1995 in a prospective cohort that reviewed more than 110,000 female nurses in the their 30 s to early 50 s who did not have Diabetes 30 years prior to the study. ${ }^{8}$ After taking age into consideration, BMI was the main factor predicting the risk of developing Diabetes Mellitus. It was noted that the risk of Diabetes surges even with minimal weight gain.

There is a direct link between the amount of visceral fat tissue surrounding the intra-abdominal viscera and insulin resistance resulting in major defects in glucose metabolism and high insulin level 
in the fasting state. ${ }^{9}$ In the Swedish Obese Subjects Study, ${ }^{10}$ Diabetes was noted to have a prevalence close to $15 \%$ among obese individuals at the start of the study. Gastric bypass leading to significant weight loss resulted in close to $70 \%$ diabetes remission rate compared with just more than $15 \%$ in the obese control group. An earlier Swedish prospective study from 1985 yielded similar conclusion. ${ }^{11}$ It reviewed around 700 middle age men who lived in Sweden designated by their same year of birth. Their anthropometric measures including waist circumference were recorded at the age of 53. Then at the age of 66, these measurements were re-examined and correlated to their risk of developing Diabetes. Those with higher waist circumference had a higher risk of developing Diabetes and the study clearly demonstrated the role of central adiposity, as a metabolic risk factor for Diabetes.

Another adverse health effect related to obesity is the risk of Gallstones. Gallstones are much more prevalent in obese individuals, especially in women with BMI greater than 40.0 in comparison with those with normal BMI. Obesity seems to lead to more than six folds increase in the risk. ${ }^{12}$ This might be to be due both to change of bile components (increased bile cholesterol level) and to delayed gall bladder emptying. Obesity is also a recognised risk factor for Non-alcoholic fatty liver disease. ${ }^{13}$ Obese individuals (mainly those with diabetes) frequently show abnormal liver test result with raised enzymatic levels, deranged lipid profile (mainly triglycerides) and liver biopsy findings of steatohepatitis. A review of liver biopsy samplings acquired from obese individuals with abnormal liver function tests, showed septal fibrosis in $30 \%$ and cirrhosis in $10 \%$ in individuals with BMI greater than 25.0. ${ }^{14}$

In addition, numerous types of cancer are more common in overweight individuals, including breast, gallbladder, ovarian, cervical and endometrial cancers in females and bowel and prostate cancers in males. ${ }^{15}$ A prospective study in 1985 examined the mortality rate in relation to overweight and cancer in more than 700 thousand individuals of both genders over more than 10 years. In comparison with individuals with BMI in the normal range, the male subjects who were more than $30 \%$ overweight had a mortality ratio for cancer of 1.33; and the female subjects 1.55 . This mortality ratio for cancer was lower than for heart attacks, diabetes and gastrointestinal diseases.

\section{Fat mass effect}

Increased fat accumulation in the soft tissues around the neck, thorax and trunk exerts adverse effect on the ability of the lungs to expand due to the mechanical restriction of thoracic wall movement and reduced contractility of the diaphragm. This in return reduces lung volumes and results in hypoventilation. In the erect position, the hypoventilation is partially compensated for. It, however, worsens significantly in the supine position. In addition, obese individuals with obstructive sleep apnoea usually have narrower upper airway passage due to increased fat deposition subcutaneously around the larynx. The combination of this in addition to mechanical restriction of thoracic wall movement and hypoventilation lead to episodic apnoeic attacks. ${ }^{16}$

Obesity at a relatively younger age can lead to increased risk of knee osteoarthritis later in life. ${ }^{17}$ This was demonstrated in a study in 1999 that reviewed the BMI of more than 1100 male medical students in their early twenties and then a few times at follow-ups. The students were assessed for the risk of developing Osteoarhritis with self-reporting symptoms and clinical and radiological evidence. High $\mathrm{BMI}$ at young age was directly linked to a higher risk of developing knee osteoarthritis at a later stage. This signifies that adverse effect of adolescent and early life obesity in the pathogenesis of osteoarthritis.
In addition to the adverse outcomes of obesity on health. Obesity also adversely affects the mental and social well-being. Sullivan et al., ${ }^{18}$ carried out an intervention study to analyse the negative effect of obesity on mental well-being. The study sample included around 800 obese men with BMI greater then 34.0 and more than 900 women with BMI more than 38.0. Patients with chronic health conditions were set as the reference group. The obese individuals evidently declared worse overall health status and lack of energy. They suffered with depression (which was more prevalent in obese women). Anxiety, low confidence and self-esteem were more common in the obese group. Moreover, the general mental status was poorer than in patients with other chronic diseases. The main contributor to this was lack or reduced physical activity. The effort related to attempting to lose weight and body image issues were significant extra risk factors. Prejudice in many cultures against obese individuals could be the driving factor for depression, anxiety and low mood. This even manifests in children in their early years of primary school. Obese individuals are discriminated against in both educational and workplace settings. ${ }^{19}$

In contrast, in cultures where obesity is not socially defamed this might not be the case. A study in Bangladesh by Asghar et al., ${ }^{20}$ that reviewed more than a thousand individuals, showed reduced Montgomery-Åsberg Depression Rating Scale (MADRS) score in overweight and obese individuals (taking age and gender into consideration) (20). This supports the hypothesis that the lack of obesity stigma in some non-westernized societies may lead to better mental wellbeing among the obese and overweight individuals. The stigma of obesity is, however, widespread worldwide. The idea of being labelled 'obese' is sometimes considered disastrous. A survey in 1997 reported that a quarter of women and one fifth of men surveyed stated they would go to a great length to avoid obesity and to be the weight they wish. ${ }^{21}$ Some women reported worries and concerns around pregnancy due to the unavoidable weight gain.

The biased message spreading in many cultures nowadays that shames obese individuals made expressing negative attitude towards overweight individuals a putative form of prejudice. Evidence has shown that discrimination against obese individuals is very common in many life settings. Several studies reported reduced employability even with similar credentials and skills. ${ }^{22}$ There is a negative perception of overweight individuals in workplace settings as they are typically considered less proficient with no self-control. ${ }^{23}$ Unfortunately, this biased discrimination against obese individual has adversely affected their income and career progression. Even in the health care service, the obesity stigma is prevalent. Negative perception and attitude towards obese patients have been recognised among healthcare staff and medical students. ${ }^{24}$

\section{Economic burden of obesity}

Obesity exerts an enormous economic burden on the already outstretched healthcare systems in many countries. It is associated with a nearly 40 percent increase in health provision spending and more than 70 percent increase in treatment spending in comparison with around 20 to $30 \%$ increase in spending due to smoking. The healthcare spending is even less for alcohol dependence. ${ }^{25}$ The intricacies of the obesity epidemic and its consistently growing list of health complications prove fully evaluating its financial burden difficult. One of the early comprehensive reviews of the health care expenses ascribed to obesity was performed in $2001 .^{26}$ Around 20 studies published in the 1990s (the majority of which were cross-sectional) proposed that obesity was responsible for close to 7 percent of the overall healthcare expenditure in the USA and an 
estimate of more than $3 \%$ of the national healthcare spending in other countries which were included in the reports. Nearly a decade later, another systematic review on the subject ${ }^{27}$ anticipated that obesity is responsible for about 0.5 percent to less than 3 percent of any country's total medical care expenditures. However, the reasons for the differences in the estimated cost and the main components of it remain uncertain. This has highlighted common flaws in literature on the subject with lack of optimal methodologically designed studies.

\section{Conclusion}

There is a concerning strident rise in the prevalence of obesity worldwide and this has made obesity a major global public health problem. Obesity is a complex condition with multifactorial aetiology. It can lead to a higher risk of early death and increased mortality overall. It is also associated with several chronic diseases due to either the mass effect of the expanding adipose tissue or the its direct metabolic effect. These including diabetes, stroke, coronary artery disease, hypertension, respiratory disease and obstructive sleep apnoea, osteoarthritis and gallstones. Obesity is also linked to many types of cancer. In addition, obesity is known to have adverse psychological and social consequences to the individuals affected. The obesity epidemic places a huge burden on national economies with its high medical care cost. The overall cost of obesity is, however, difficult to evaluate and this emphasises the urgent need for standardised methodologically designed studies to assess the situation. In addition, across-the-board local and international strategic plans are required for prevention and control of this epidemic.

\section{Acknowledgments}

The author declares no conflict of interest. The author would like to thank all the researchers in the field of obesity and weight management.

\section{Conflicts of interest}

There are no conflicts of interest.

\section{Funding}

None.

\section{References}

1. World Health Organization. Fact sheet: obesity and overweight. 2019.

2. Wilding J. Are the causes of obesity primary environmental? Yes. BMJ. 2012;345:e5843.

3. Bray G. Medical Consequences of Obesity. The Journal of Clinical Endocrinology \& Metabolism. 2004;89(6):2583-2589.

4. Adams KF, Schatzkin A, Harris TB, et al. Overweight, obesity, and mortality in a large prospective cohort of persons 50 to 71 years old. $N$ Engl J Med. 2006;24:355(8):763-778.

5. Jee SH, Sull JW, Park J, et al. Body-mass index and mortality in Korean men and women. $N$ Engl J Med. 2006;355(8):779-787.

6. Huang $\mathrm{z}$, Willett WC, Manson JE, et al. Body weight, weight change, and risk for hypertension in women. Ann Intern Med. 1998;128(2):81-88.

7. Hubert HB, Feinleib M, McNamara PM, et al. Obesity as an independent risk factor for cardiovascular disease: a 26-year follow-up of participants in the Framingham Study. Circulation. 1983;67(5):968-977.
8. Colditz GA, Willett WC, Rotnitzky A, et al. Weight gain as a risk factor for clinical diabetes mellitus in women. Ann Intern Med. 1995;122(7):481486.

9. Caprio S. Relationship between abdominal visceral fat and metabolic risk factor. AM J Hum Biol. 1999;11(2):259-266.

10. Siöström C, Lissner L, Siostrom L. Relationships Between Changes in Body Composition and Changes in Cardiovascular Risk Factors: The SOS Intervention Study. Obesity Research. 1997;5(6):519-530.

11. Ohlson LO, Larsson B, Svardsudd K, et al. The influence of body fat distribution on the incidence of diabetes mellitus.13.5 years of follow up of the participants in the study of men born in 1913. Diabetes. 1985;34(10):1055-1058.

12. Stampfer MJ, Maclure KM, Colditz GA, et al. Risk of symptomatic gallstones in women with severe obesity. Am J Clin Nutr. 1997;55(3):652658.

13. Sheth SG, Gordon FD, Chopra S. Nonalcoholic steatohepatitis. Ann Intern Med. 1997;126(2):137-145.

14. Ratziu V, Giral $\mathrm{P}$, Charlotte $\mathrm{F}$, et al. Liver fibrosis in overweight patients. Gastroenterology. 2000;118(6):1117-1123.

15. Garfinkel L. Overweight and cancer. Ann Intern Med. 1985;103(6 (Pt 2)):1034-1036.

16. Grunstein RR. Pulmonary function, sleep apnoea and obesity. Clinical Obesity. In: Kopelman PG, Stock MJ, editors. Oxford. 1998. p. 248-289.

17. Gelber AC, Hochberg MC, Mead LA, et al. Body mass index in young men and the risk of subsequent knee and hip osteoarthritis. Am J Med. 1999; 107(6):542-548

18. Sullivan M, Karlsson J, Sjostrom L, et al. Swedish obese subjects (SOS) - an intervention study of obesity. Baseline evaluation of health and psychosocial functioning in the first 1743 subjects examined. Int $J$ Obes Relat Metab Disord. 1993;17(9):503-512.

19. Wadden TA, Stunkard AJ. Social and psychological consequences of obesity. Ann Intern Med. 1985;103(6(Pt 2)):1062-1067.

20. Ashgar S, Magnusson A, Khan A, et al. Retracted: In Bangladesh, overweight individuals have fewer symptoms of depression than nonoverweight individuals. Obesity research journal. 2010;18(6):1143-1145.

21. Garner DM. The body image survey results. Psychol Today. 1996;30:3047.

22. Roehling MV. Weight-based discrimination in employment: psychological and legal aspects. Pers Psychol. 1999; 52(4):969-1017.

23. Paul RJ, Townsend JB. Shape up or ship out? Employment discrimination against the overweight. Employee Responsibilities Rights Journal. 1995;8(2):133-145.

24. H J Wiese, J F Wilson, R A Jones, et al. Obesity stigma reduction in medical students. Int J Obesity. 1992;16(11):859-868.

25. Sturm R. The effects of obesity, smoking, and drinking on medical problems and costs. Health Aff (Millwood). 2002;21(2):245-253.

26. Thompson D, Wolf AM. The medical-care cost burden of obesity. Obes Rev. 2001;2:189-197.

27. Withrow D, Alter DA. The economic burden of obesity worldwide: a systematic review of the direct costs of obesity. Obesity reviews. 2011;12(2):131-141. 